\title{
Stat3 mediates myeloid cell-dependent tumor angiogenesis in mice
}

\author{
Maciej Kujawski, ${ }^{1}$ Marcin Kortylewski, ${ }^{1}$ Heehyoung Lee, ${ }^{1}$ Andreas Herrmann,, \\ Heidi Kay, ${ }^{2}$ and Hua Yu'
}

${ }^{1}$ Beckman Research Institute, City of Hope National Medical Center, Duarte, California, USA.

2University of South Florida College of Public Health, Tampa, Florida, USA.

\begin{abstract}
The underlying molecular mechanisms that cause immune cells, mediators of our defense system, to promote tumor invasion and angiogenesis remain incompletely understood. Constitutively activated Stat 3 in tumor cells has been shown to promote tumor invasion and angiogenesis. Therefore, we sought to determine whether Stat3 activation in tumor-associated inflammatory cells has a similar function. We found that Stat3 signaling mediates multidirectional crosstalk among tumor cells, myeloid cells in the tumor stroma, and ECs that contributes to tumor angiogenesis in mice. Myeloid-derived suppressor cells and macrophages isolated from mouse tumors displayed activated Stat 3 and induced angiogenesis in an in vitro tube formation assay via Stat 3 induction of angiogenic factors, including VEGF and bFGF. Stat3-regulated factors produced by both tumor cells and tumor-derived myeloid cells also induced constitutive activation of Stat 3 in tumor endothelium, and inhibiting Stat3 in ECs substantially reduced in vitro tumor factor-induced endothelial migration and tube formation. In vivo assays demonstrated the requirement for Stat 3 signaling in tumorassociated myeloid cells for tumor angiogenesis. Our results indicate that, by virtue of the ability of Stat 3 in tumor cells and tumor-derived myeloid cells to upregulate expression of factors that activate Stat 3 in ECs, Stat 3 mediates multidirectional crosstalk among tumor cells, tumor-associated myeloid cells, and ECs that contributes to tumor angiogenesis.
\end{abstract}

\section{Introduction}

Despite the emerging role of immune cells in mediating carcinogenesis/tumor angiogenesis, the very fundamental function of our immune system as a defense mechanism against pathogens is clear. An essential part played by immune cells as an extrinsic tumor suppressor has also been well demonstrated $(1,2)$. However, it is evident that there is a general lack of $\mathrm{CD}^{+} \mathrm{T}$ cells infiltrating tumor and a prominent presence of $\mathrm{T}$ regulatory cells and myeloid-derived suppressor cells (MDSCs) in the tumor stroma, promoting tumor immune evasion (3-7). Several recent studies also underscore the importance of the interplay between immune suppression and angiogenesis mediated by tumor-associated myeloid cells $(8,9)$. Evidence supporting this includes the observations that tumor-associated MDSCs and/or tumor-associated macrophages (TAMs) produce angiogenic factors, MMP9, VEGF, and IL-1 $\beta$, which enhance MDSC accumulation in tumors and contribute not only to tumor immune suppression, but also to tumor angiogenesis (10-15). A similar role of TAMs in promoting tumor immune evasion and angiogenesis/evasion has also been recognized (16-18). In spite of this, how tumor cells might subvert the immune system from performing its antitumor functions to promoting immunosuppression and tumorigenesis has been intriguing and equivocal.

Stat 3 is a point of convergence for numerous oncogenic signaling pathways frequently activated in cancer (19-21). Stat3 is constitutively activated with high frequency in cancer and is well known for promoting tumor cell survival and proliferation (19). Recent studies from our group as well as other laboratories suggest that Stat 3 is an

Nonstandard abbreviations used: MDSC, myeloid-derived suppressor cell; TAM, tumor-associated macrophage.

Conflict of interest: The authors have declared that no conflict of interest exists. Citation for this article: J. Clin. Invest. 118:3367-3377 (2008). doi:10.1172/JCI35213. important mediator of tumor immune suppression. Stat 3 not only inhibits expression of numerous Th1 immunostimulatory molecules, such as IL-12, IFNs, and costimulatory molecules necessary for inducing antitumor immunity, but also promotes expression of a number of immunosuppressive factors, many of which in turn activate Stat 3 in various immune cells in the tumor stroma and/or tumor draining lymph nodes $(3,22,23)$. Among the immunosuppressive factors that are inducible by Stat 3 activity are IL-10, VEGF $(24,25)$, and IL-23 (M. Kortylewski, unpublished observations). Recent studies reveal that these 3 factors exert immune suppressive effects, at least in part, by activating Stat 3 in various immune cells $(3,26)$. This propagation of Stat 3 activity from tumor cells to immune cells allows a crosstalk from tumor cells to various stromal cells, leading to tumor immune suppression $(3,27)$. Of note, a number of the immunosuppressive factors, such as VEGF, produced by tumor cells in a Stat3-dependent manner are also angiogenic factors. A role of constitutively activated Stat 3 in tumor cells in promoting tumor angiogenesis and metastasis has been documented $(25,28-30)$. A key unanswered question is whether Stat 3 activation in tumor-associated diverse inflammatory cells is critical for tumor angiogenesis. Our current study shows that Stat 3 activity in tumor MDSCs, as well as TAMs, is critical for their angiogenic potential. We identified that Stat 3 activity in tumor-derived myeloid cells upregulated the expression of a number of known Stat 3 target genes that are angiogenic, including VEGF, bFGF, and MMP9. Antibody neutralization experiments showed that both VEGF and bFGF contributed to myeloid cell-mediated, Stat3-dependent angiogenesis. In addition, CCL2, CXCL2, and IL-1 $\beta$, which have previously been shown to contribute to myeloid cell-mediated angiogenesis (31, $32)$, required Stat3 for their elevated expression in tumor-derived myeloid cells. Our results suggest that Stat 3 activity in immune cells can contribute to tumor angiogenesis at multiple levels. 


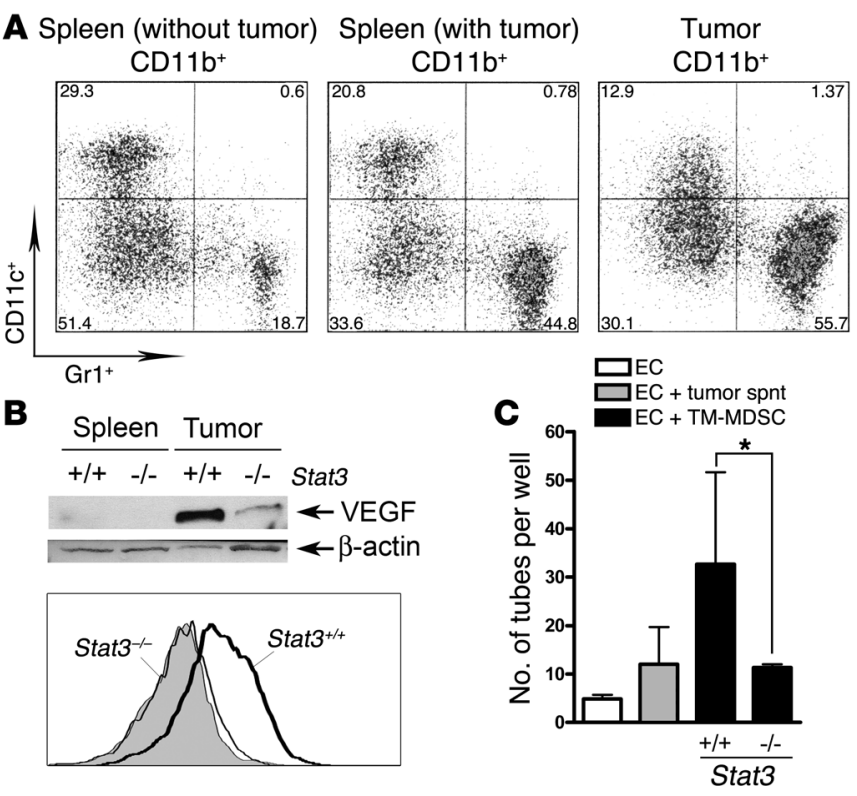

We have previously demonstrated that Stat 3 activity induces production of pleiotropic factors that inhibit DC functional maturation (23). These factors inhibit DC maturation through Stat3 activation in differentiated, yet immature, DCs $(3,23)$. Thus, inhibition of antitumor immunity involves a cascade of Stat 3 activation propagating from tumor cells to DCs. Based on these findings, we further address whether a similar cascade of Stat 3 signaling from tumor cells and tumor-derived myeloid cells to ECs mediates tumor angiogenesis. The current study shows that Stat 3 was constitutively activated in tumor endothelium and that activation of Stat 3 in ECs depended on factors regulated by Stat 3 in both tumor cells and tumor-derived myeloid cells. Our results also show that Stat 3 activation in ECs was required for tumor factor-induced tube formation and migration. In vivo analyses further demonstrated a role of Stat 3 in mediating tumor-derived myeloid cells in tumor angiogenesis. The present study underscores the importance of Stat 3 in mediating a multidirectional crosstalk among tumor cells, tumor stromal myeloid cells, and ECs and suggests that tumor immune suppression and angiogenesis are tightly interwoven and mediated, at least in part, by Stat 3 .

\section{Results}

Role of Stat 3 in tumor-associated MDSCs and tumor angiogenesis. We first assessed the relative presence of $\mathrm{CD} 11 \mathrm{~b}^{+} \mathrm{Gr} 1^{+} \mathrm{CD} 11^{-} \mathrm{MDSC}$ compared with $\mathrm{CD} 11 \mathrm{~b}^{+} \mathrm{Gr} 1^{-} \mathrm{CD} 11 \mathrm{c}^{+}$DCs in B16 melanoma tumors. Tumor and spleen tissues were harvested from C57BL/ 6 naive mice and mice bearing large B16 melanoma tumors approximately $10 \mathrm{~mm}$ in diameter. Flow cytometric analysis of CD11 $\mathrm{b}^{+}$myeloid cells enriched from spleen and tumor tissues indicated that while the percentage of DCs remained similar in normal spleens and spleens derived from tumor-bearing mice, the number of MDSCs in spleens from tumor-bearing mice drastically increased (Figure 1A). $\mathrm{CD} 11 \mathrm{~b}^{+} \mathrm{Gr} 1^{+} \mathrm{CD} 11^{-} \mathrm{MDSC}$ were the largest population of myeloid cells residing within tumor tissue. In contrast, the number of tumorassociated DCs was small, and these were less phenotypically distinct (Figure 1A). We have previously found that tumor-infiltrating $\mathrm{Gr}^{+}$ cells, including MDSCs, increase Stat 3 activity relative to their splenic

\section{Figure 1}

Role of Stat3 in the contribution of tumor-associated MDSCs to tumor angiogenesis. (A) Flow cytometric analysis of cells isolated from spleens and B16 tumors shows accumulation of $\mathrm{Gr} 1+\mathrm{CD} 11 \mathrm{~b}+$ cells in the tumors and spleens of tumor-bearing mice. Cells were labeled with antibodies for CD11b, CD11c, and Gr1, as indicated. (B) Top: Western blot analysis of VEGF protein levels in $\mathrm{Gr} 1{ }^{+} \mathrm{CD} 11 \mathrm{~b}+$ cells isolated from B16 melanoma. Gr1+CD11b+ cells were purified by flow cytometric cell sorting, using spleens and/or tumors from mice with Stat $3^{+/+}$and Stat3-/hematopoietic systems. Bottom: Flow cytometric analysis of CD11 $\mathrm{b}^{+}$ cells isolated from B16 tumors indicates increased Stat3 activation in Stat $3^{+/+}$but not in Stat3 ${ }^{-/-}$cells. (C) ECs form tube structures when

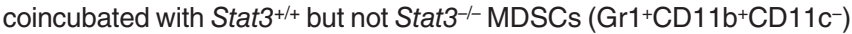
isolated directly from B16 melanoma (tumor-associated MDSCs; TMMDSC). Tumor supernatant (spnt) was also added to the EC culture as a positive control. Graph shows mean \pm SEM of 2 independent experiments $(n=3) .{ }^{*} P<0.05$. counterparts (3). Because Stat 3 regulates the expression of several proangiogenic factors, including VEGF, in tumor cells $(25,29)$, we next determined whether MDSCs are involved in promoting tumor vascularization. We used conditional and inducible Stat 3 knockout (Stat $3^{\text {flox}} / \mathrm{Mx}$-Cre $)$ mice to abrogate Stat 3 signaling in all hematopoietic cell lineages. As previously reported $(3,33)$, repeated treatment with poly(I:C) allows for functional deletion of Stat 3 in hematopoietic cells in adult Stat $3^{f l o x} / \mathrm{Mx}$-Cre mice. Cell suspensions prepared from tumors or spleens of mice with Stat3-positive and Stat3-negative hematopoietic systems were sorted by fluorescence-activated cell sorting to isolate MDSCs. Results of Western blot analysis indicated that whereas splenic $\mathrm{CD} 11 \mathrm{~b}^{+} \mathrm{Gr} 1^{+} \mathrm{CD} 11 \mathrm{c}^{-}$MDSCs did not produce a detectable amount of VEGF, tumor-associated Stat $3^{+/+}$MDSCs, but not Stat $3^{-/-}$ MDSCs, expressed markedly more VEGF (Figure 1B). Flow analysis of the whole $\mathrm{CD} 11 \mathrm{~b}^{+}$population within the tumor tissue indicated high Stat 3 activation only in the $S t a t 3^{+/+}$cells (Figure 1B).

To directly test whether Stat 3 activity contributes to angiogenesis mediated by tumor-associated MDSCs, we performed EC tube formation assay using collagen matrix. As a positive control to show responsiveness of the cultured ECs, we used conditioned medium containing $2.5 \%$ of culture medium from C4 melanoma cells. For comparison, ECs were cocultured together with MDSCs freshly isolated from growing B16 tumors. As shown in Figure $1 \mathrm{C}$, Stat $3^{+/+}$tumor-associated MDSCs induced EC tube formation. In contrast, the ability of tumor-derived Stat $3^{-/-}$MDSCs to promote EC function was markedly compromised. The residual tube formation on collagen matrix induced by Stat3-deficient MDSCs may reflect the involvement of other signaling pathways in promoting angiogenesis $(34,35)$.

Stat 3 activation and tumor angiogenesis is modulated by other myeloid cells. Our results above underline an important role of Stat 3 signaling in MDSCs for promoting tumor angiogenesis. Coincubation of splenic Stat $3^{+/+}$MDSCs with ECs in the presence of a relatively low concentration of tumor-conditioned medium resulted in a 3 -fold increase in the number of tube-like structures formed by ECs on collagen matrix (Figure 2A). Surprisingly, even in the absence of tumor-derived factors, the addition of MDSCs enhanced the 


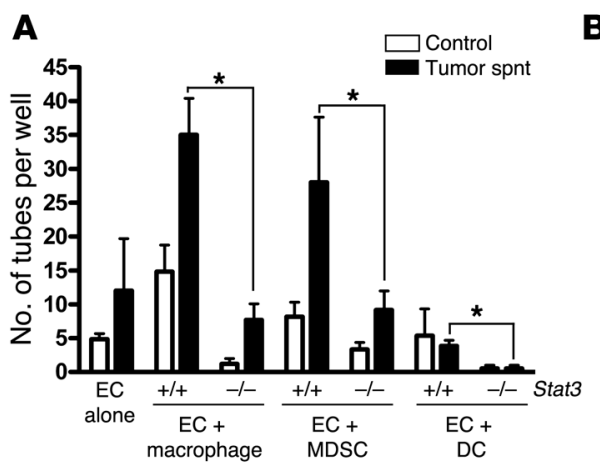

C

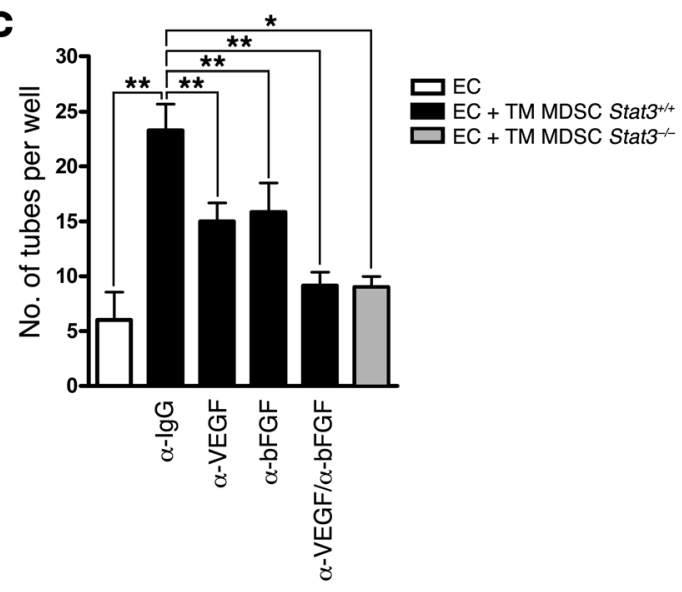

B

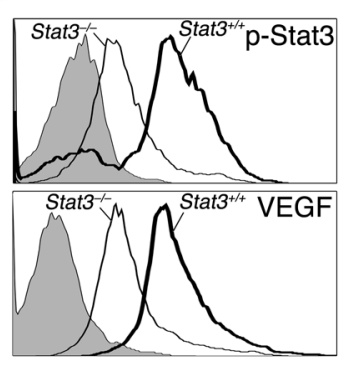

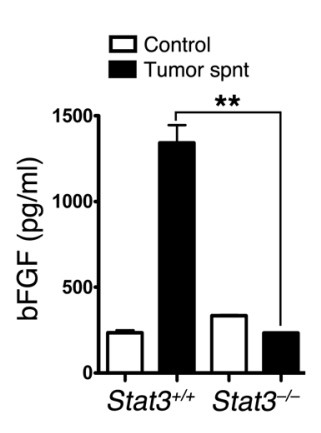

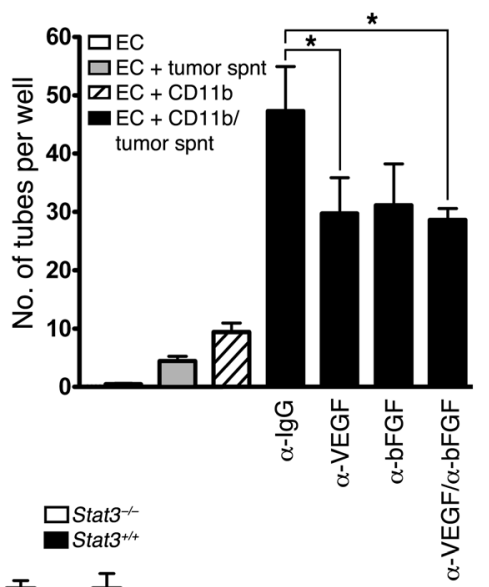

D

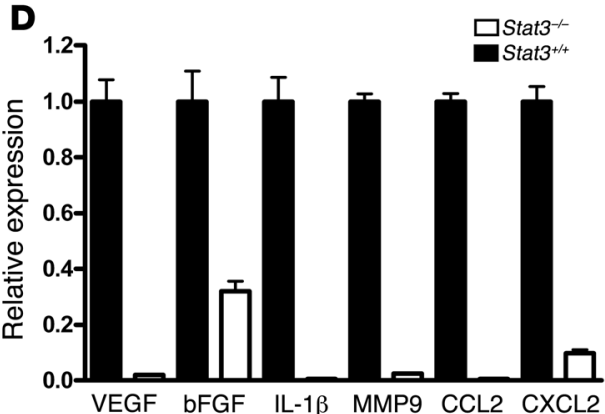

Figure 2

Role of Stat3 activity and different populations of myeloid cells in angiogenesis. (A) In vitro collagen tube formation assays using different subsets of $\mathrm{CD} 11 \mathrm{~b}^{+}$myeloid cells. Both Gr1 ${ }^{+} \mathrm{CD} 11 \mathrm{~b}+\mathrm{CD} 11 \mathrm{c}^{-}$cells and Gr1-CD11b+CD11 $\mathrm{c}^{-}$cells in the presence of $2.5 \%$ tumor-conditioned medium successfully induced ECs to form tube-like structures. Gr1-CD11b+CD11c+ DCs did not induce efficient EC tube formation. Graph shows mean \pm SEM of 2 independent experiments. (B) VEGF and bFGF expression in CD11 $\mathrm{b}^{+} \mathrm{Gr} 1^{+}$myeloid cells contribute to angiogenesis. Splenic

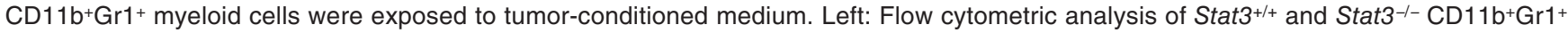
myeloid cells isolated from spleens of tumor-bearing mice for phospho-Stat3 (p-Stat3) and VEGF levels. Levels of bFGF in the indicated cells were determined by the Luminex system (see Methods). Graph shows mean \pm SEM of 2 independent experiments. Right: Neutralization of VEGF and VEGF together with bFGF partially but significantly reduced myeloid cell-mediated angiogenesis. Graph shows mean \pm SEM of 2 independent experiments with triplicates. (C) VEGF and bFGF are important for Stat3-dependent, tumor-derived MDSC-mediated angiogenesis. MDSCs purified from B16 tumors were used for tube formation assays in the presence of the indicated antibodies. Data are mean \pm SEM of 3 independent experiments, each involving 10-15 pooled mice per group done in triplicates. (D) Stat3 activity in tumor-associated myeloid

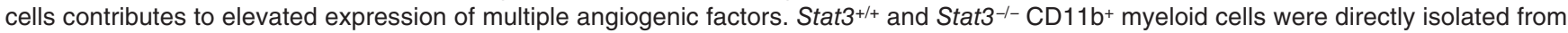
B16 tumors, and real-time RT-PCR was performed to detect RNA levels of the indicated genes. RNA levels of each indicated gene relative to a housekeeping gene (either 18s or GAPDH) in Stat $3^{+/+}$myeloid cells were assigned as 1. Data are mean \pm SEM $(n=3) .{ }^{*} P<0.05 ;{ }^{* \star} P<0.01$.

number of tubes, possibly as a result of low-level Stat 3 activation induced by growth factor-rich culture medium. ECs incubated in the presence of Stat $3^{-/-}$MDSCs with or without tumor-conditioned medium formed fewer tubes than did the respective controls. Similar, although slightly weaker, proangiogenic effects were observed when ECs were coincubated with the fraction of non-MDSC, nonDC myeloid cells (CD11 $\mathrm{b}^{+} \mathrm{Gr} 1^{-} \mathrm{CD} 11 \mathrm{c}^{-}$) composed mostly of macrophages. Moreover, ablating Stat 3 completely blocked the proangiogenic effects of CD $11 \mathrm{~b}^{+} \mathrm{Gr} 1^{-} \mathrm{CD} 11 \mathrm{c}^{-}$cells on ECs. In contrast, splenic $\mathrm{Stat}^{+/+}$DCs did not stimulate tube formation by ECs. Furthermore, ablating Stat3 in DCs had some inhibitory effect on EC function, resulting in abrogation of tube formation (Figure $2 \mathrm{~A}$ ). Next, we investigated whether splenic MDSCs, which show relatively low levels of Stat3 phosphorylation, acquire proangiogenic potential as a result of induced Stat 3 activation in the presence of tumor-secreted factors. Flow analysis indicated that after stimulation with tumor-con- ditioned medium, MDSCs isolated from spleens of tumor-bearing Stat $3^{+/+}$mice showed increased levels of Stat 3 phosphorylation and VEGF expression compared with their Stat $3^{-/-}$counterparts (Figure 2B). Furthermore, we observed Stat3-dependent induction of proangiogenic bFGF in myeloid cells isolated from spleens of tumorbearing mice stimulated with tumor-conditioned medium (Figure $2 \mathrm{~B})$. To assess the role of these 2 factors in myeloid cell-induced, Stat3-mediated angiogenesis, we performed antibody neutralization experiments. Myeloid cells isolated from spleens were exposed to low levels of tumor-conditioned medium (i.e., 2.5\%) before they were used for tube formation assay in the presence of specific antibodies. We found partial but significant reduction in the number of tubes after coincubation with anti-VEGF antibody and the combination of both anti-VEGF and anti-bFGF antibodies (Figure 2B).

Results from the in vitro experiments using tumor supernatant suggested that Stat3 downstream factors, including VEGF 
A

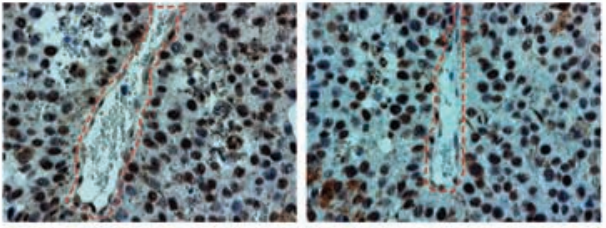

B
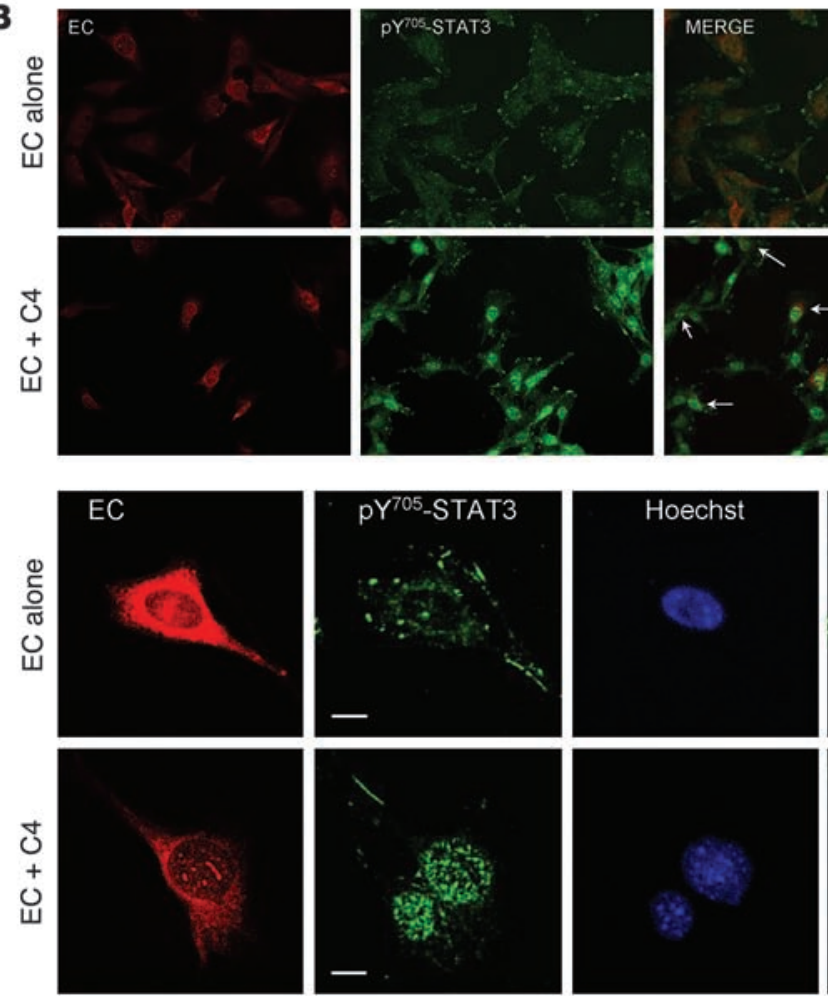
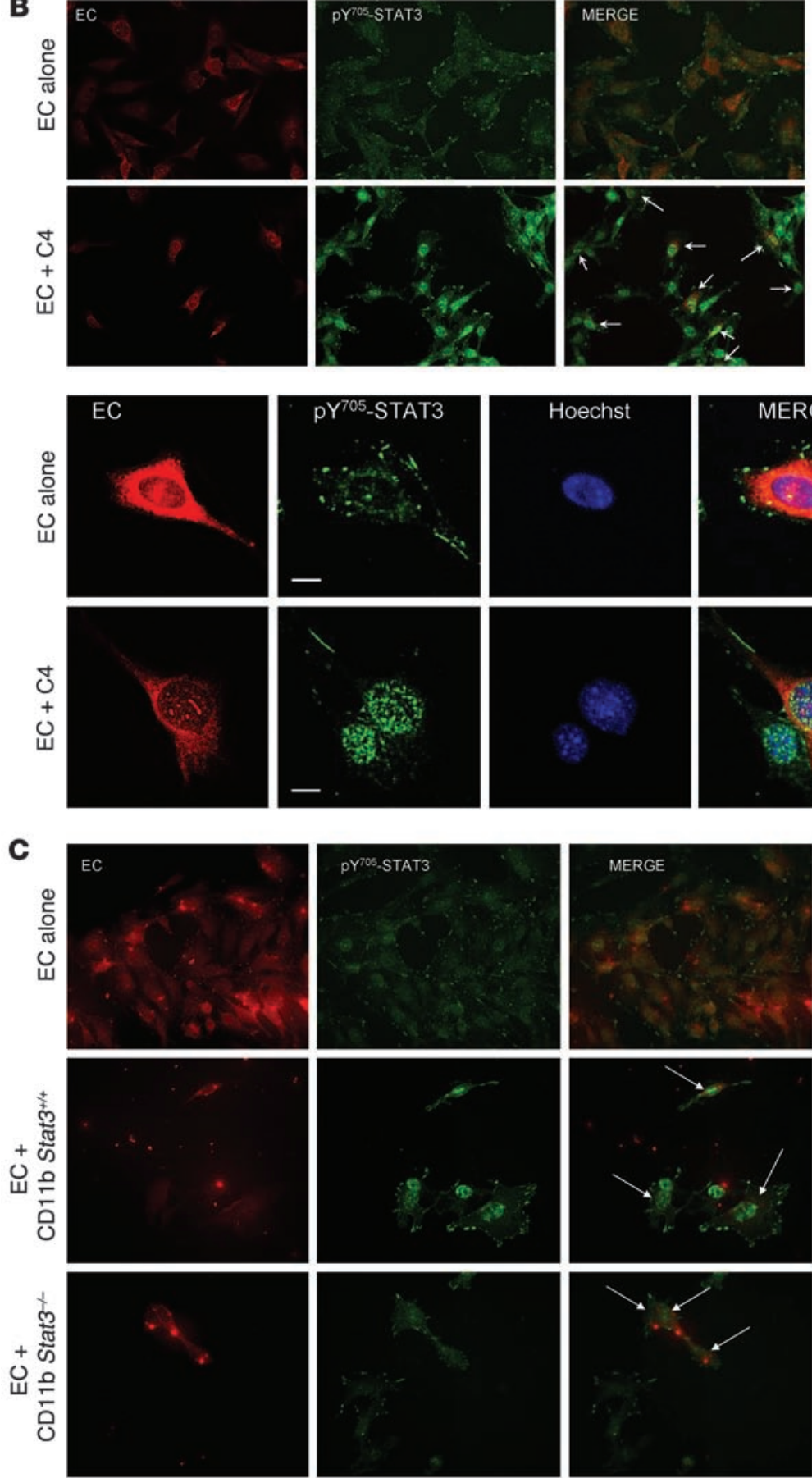
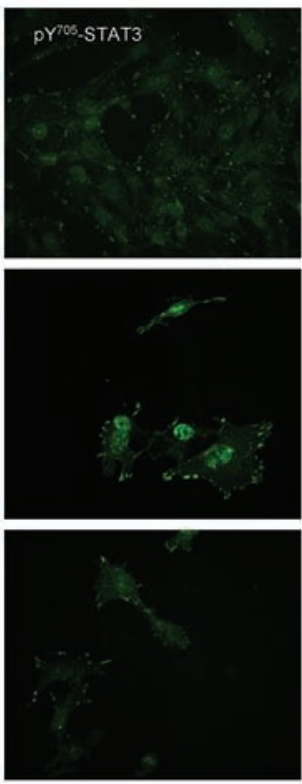
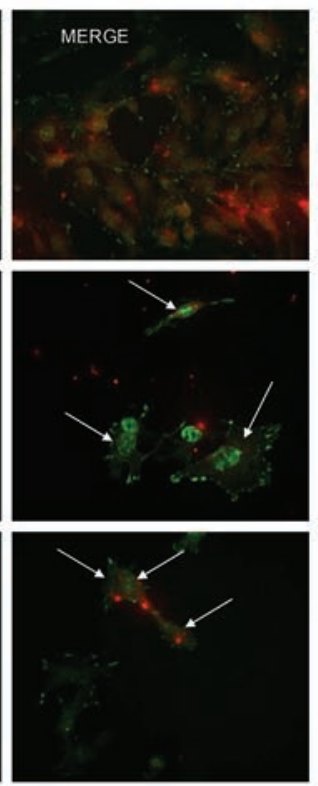

Figure 3

Stat3 is constitutively activated in ECs exposed to the tumor milieu in vivo and in vitro. (A) Immunohistochemistry staining of mouse B16 melanoma tissue sections with $\mathrm{pY}^{705}$-Stat3 antibody. Dashed lines indicate tumor blood vessels. (B) Immunofluorescent staining ( $\mathrm{pY}^{705}$-Stat3 and goat anti-rabbit Alexa Fluor 488 antibodies) of ECs cultured alone or with mouse C4 melanoma cells at a 1:5 ratio. ECs were labeled with CellTracker Orange (red) prior to coculturing. Arrows indicate ECs. Below are examples of nuclear translocation of phospho-Stat3 in cells from the same experiment visualized with confocal microscopy. (C) Tumor-derived CD11 b $\mathrm{b}^{+}$myeloid cells induce Stat3 activation in ECs. Stat $3^{+/+}$ and Stat3 ${ }^{-/-}$CD $11 b^{+}$myeloid cells isolated from B16 tumors were added to the EC culture. ECs were labeled with CellTracker Orange (red), and pY705-Stat3 was detected using rabbit antibody and goat anti-rabbit Alexa Fluor 488 antibody (green). Arrows indicate ECs. (D) Stat3-positive myeloid cells interact with ECs in human breast cancer tissues. Human breast cancer tissue sections were first stained with rabbit $\mathrm{pY}^{705}$-Stat3 antibody and mouse anti-CD68 antibody to detect human myeloid cells, followed by staining with secondary antibodies goat anti-rabbit Alexa Fluor 488 (green) for Stat3 and goat anti-mouse Alexa Fluor 555 (red) for CD68. Insets show higher magnification of the boxed region, both nuclear counterstaining (Hoechst 33342, blue) and CD68 staining (red). Arrows indicate CD68 ${ }^{+}$ cells; arrowhead indicates blood vessel. Original magnification, $\times 400$ (A; B top; C; and D); $\times 1,000$ (B, bottom); $\times 1,250$ (D, insets). and bFGF, contribute to tumor-associated, myeloid-mediated angiogenesis. To validate these observations under more physiological conditions, we used MDSCs directly isolated from tumors. Treating Stat $3^{+/+}$tumor-derived MDSCs with antibod-
D

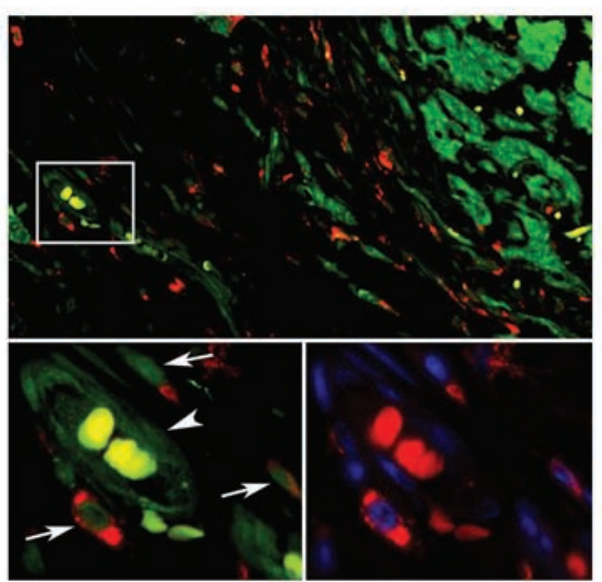




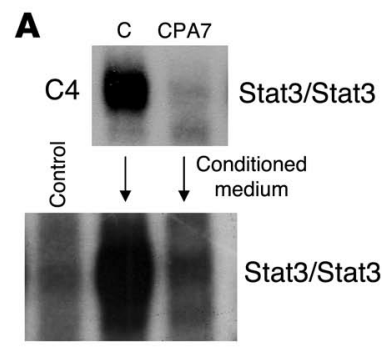

Prostate ECs
B

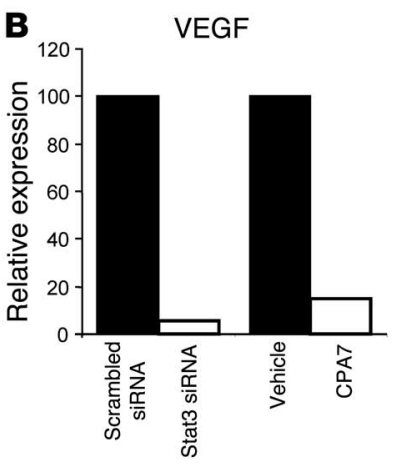

D

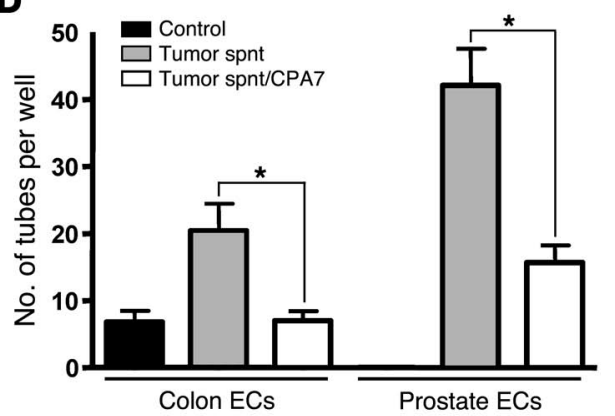

C

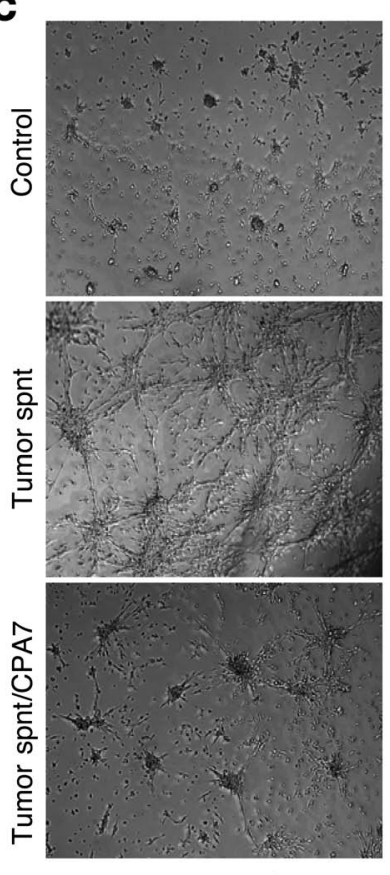

Prostate EC

\section{Figure 4}

Stat3 activity in tumor cells affects Stat3 activity and tube formation potential in ECs. (A) Tumor-conditioned media generated after blocking Stat3 activity in mouse C4 melanoma cells reduced Stat3 DNA binding in ECs, as shown by EMSA. C, DMSO control. (B) Inhibition of VEGF expression in mouse C4 melanoma cells by transfection with siRNA or Stat3 inhibitor CPA7, as detected by real-time PCR. (C) Collagen tube formation assay of ECs exposed to tumor-conditioned medium containing tumor supernatant collected from C4 melanoma cells exposed to vehicle or CPA7. The control cells were cultured in RPMI medium with 1\% FBS. Shown are representative microphotographs of 3 independent experiments. Original magnification, $\times 100$. (D) Quantification of tube-like structures per well of 48-well plates for EC lines derived from colon and prostate exposed to tumor-conditioned media. Data are mean \pm SEM of 3 independent experiments. ${ }^{\star} P<0.05$.

To determine whether Stat3 activity in tumor-infiltrating myeloid cells affects expression of other angiogenic factors in addition to VEGF, we performed quantitative real-time PCR. Results from these analyses showed that Stat 3 activity in tumor-associated myeloid cells contributed to elevated gene transcription of not only VEGF and bFGF, but also IL-1 $\beta$, MMP9, CCL2, and CXCL2 (Figure 2D). All of these molecules have been recently demonstrated to be important for MDSC- and/or TAM-induced tumor angiogenesis $(9,18,36,37)$.

Stat 3 is constitutively activated in tumor ECs in vivo and in vitro. Our results above demonstrated that Stat 3 signaling within MDSCs and in TAMs is critical for tumor angiogenesis. The next key question we addressed was how these cells communicate with ECs, thereby leading to angiogenesis. Because many of the Stat 3 target genes encoding factors - including VEGF, IL-6, bFGF, and HGF - are activators of Stat3, we hypothesized that Stat 3 signaling intrinsic to ECs is critical for their communication with tumor stromal myeloid cells. To test this hypothesis, we performed immunohistochemical staining for phospho-Stat3 in mouse B16 melanoma samples. We found that Stat 3 not only was highly activated in tumor cells, but was also persistently activated in the ECs within tumor vasculature (Figure 3A). Together with the data shown in Figure 2A, these findings suggest that Stat 3 is constitutively activated in tumor cells, in tumor myeloid cells, and in ECs. We next sought to determine whether EC Stat 3 activation is caused mainly by factors encoded by Stat 3 downstream genes, using tumor cell/EC coculture experi- ments. ECs derived from mouse prostate (38) were labeled with CellTracker OrangeCMTMR reagent (see Methods). In this experiment, ECs were mixed with $\mathrm{C} 4$ melanoma cells at a 1:5 ratio. In the absence of tumor cells or tumor factors, the ECs did not exhibit detectable Stat 3 activity, as assessed by phospho-Stat3 (pY705-Stat3) antibody staining. However, upon coculturing with C4 melanoma tumor cells, which display highly activated Stat3 (39), the ECs acquired constitutively activated Stat 3 , as demonstrated by positive nuclear staining with $\mathrm{PY}^{705}$-Stat3 antibody (Figure 3B).

To assess whether factors secreted by tumor-derived myeloid cells can directly activate Stat 3 in ECs, we added CD11 b ${ }^{+}$myeloid cells freshly isolated from B16 tumors to cultured ECs, which were labeled with CellTracker OrangeCMTMR reagent. After coincubation with tumor-derived myeloid cells, the cultured cells were stained with pY $^{705}$-Stat 3 antibody to detect Stat 3 activity in the ECs. Our results suggest that tumor-associated myeloid cells, like tumor cells, are able to produce factors that stimulate Stat 3 in ECs (Figure 3C). Using Stat $3^{-/-}$myeloid cells derived from growing B16 tumors, we further demonstrated that production of myeloid cellsecreted factors critical for stimulating Stat 3 in ECs required Stat 3 (Figure 3C). To confirm the potential importance of Stat3 signaling in myeloid cells in human cancer angiogenesis, we performed immunofluorescence staining of human breast carcinoma tissues. In 10 analyzed tissue samples, 7 showed visible infiltration of $\mathrm{CD}^{+} 8^{+}$ macrophages and other myeloid cells. We found blood vessels in 3 of the tissue samples, all of which had myeloid cells in the vessel areas 
A
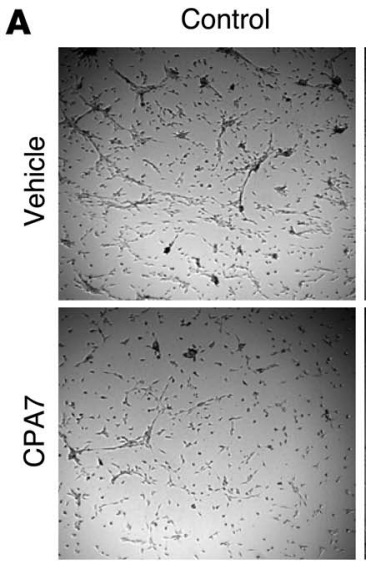

\section{B}
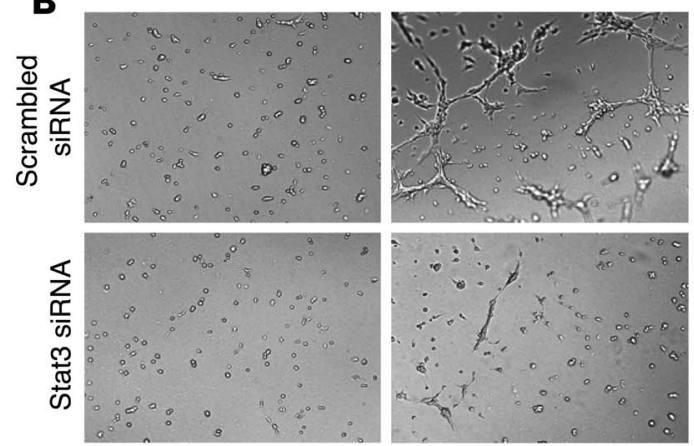

Tumor spnt
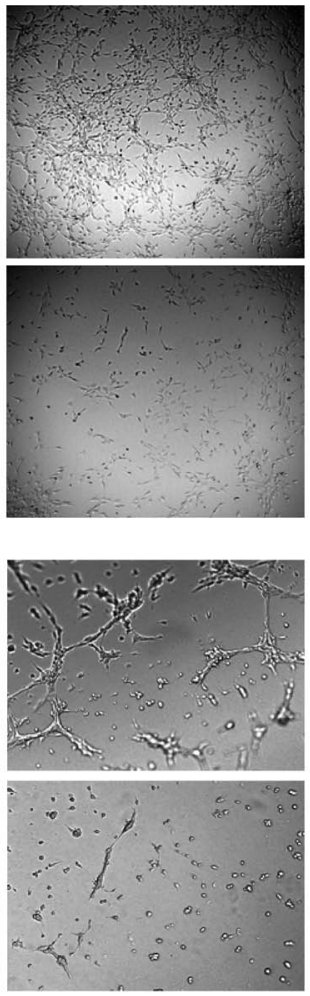
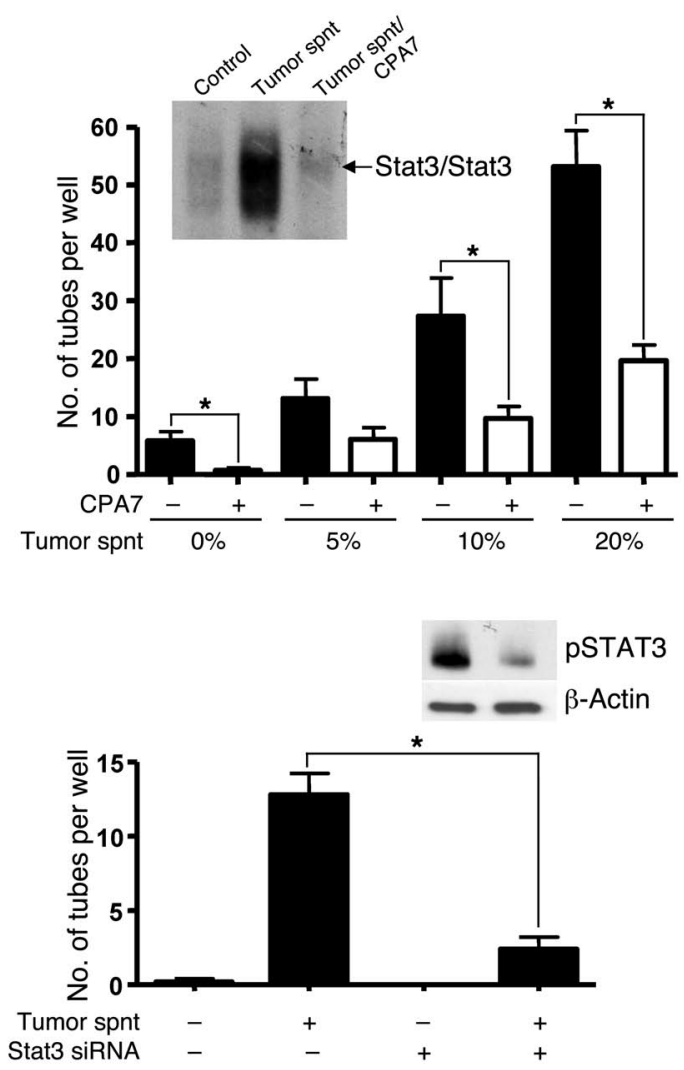

Figure 5

Stat3 signaling in ECs is important for tumor factor-induced tube formation. (A) Representative microphotographs of tube formation assay of ECs treated with vehicle or Stat3 inhibitor CPA7 for $16 \mathrm{~h}$ in the presence of control (RPMI medium with $1 \% \mathrm{FBS}$ ) or $10 \%$ tumor-conditioned media. Quantification of tube-like structures per well (48-well plate) exposed to control medium or 3 different concentrations of tumor-conditioned media with or without Stat3 inhibitor treatment is shown. Data are mean \pm SEM of 3 independent experiments. EMSA results of Stat3 DNA-binding of nuclear extracts isolated from ECs are also shown. (B) Representative microphotographs of tube formation assay of ECs transfected with scrambled or Stat3 siRNA and exposed to control (RPMI medium with 1\% FBS) or 10\% tumor-conditioned media. Quantification of tube-like structures per well (48-well plate) exposed to control medium or $10 \%$ tumor-conditioned media is shown. Data are mean \pm SEM of 3 independent experiments. Transfection efficiency, as shown by Western blot analysis of total protein lysates using indicated antibodies, is also shown. ${ }^{*} P<0.05$. Original magnification, $\times 40(\mathbf{A}) ; \times 100(\mathbf{B})$.

to varying extents. Not only tumor cells and ECs were positive for phospho-Stat3, so were $\mathrm{CD}^{+} 8^{+}$myeloid cells (Figure 3D).

EC tube formation involves not only extrinsic, but also intrinsic, Stat3 activation. We further investigated whether Stat 3 signaling intrinsic to ECs is critical for tumor angiogenesis. While tumor cell-conditioned medium induced Stat 3 DNA binding activity in ECs, inhibition of Stat 3 activity in tumor cells by transfecting siRNA or incubating with small-molecule Stat3 inhibitor CPA7 (3) in tumor cells reduced their ability to induce Stat 3 activity in ECs (Figure 4A). Because one of the Stat 3 downstream genes important for angiogenesis produced by tumor cells is VEGF, we assessed whether VEGF expression is Stat 3 dependent in C4 tumor cells. Blocking Stat3 by siRNA or CPA7 strongly decreased expression of VEGF in C4 melanoma cells (Figure 4B). When the EC Stat3 activity was high, which can be induced by soluble factors secreted by tumor cells with high Stat 3 activity, ECs formed extensive tubes on collagen matrix. In contrast, tube formation was significantly reduced when ECs displayed little Stat 3 activity because they were exposed to the tumor-conditioned medium collected from the melanoma cells treated with the Stat 3 inhibitor (Figure 4, C and D). These results, together with the findings that Stat 3 was constitutively activated in tumor endothelium, indicate that Stat 3 activation in ECs is important for angiogenesis.

We therefore tested whether blocking Stat 3 activity in ECs affects the cells' angiogenic functions. Treating the mouse ECs with a small-molecule Stat 3 inhibitor, CPA7, inhibited Stat3 DNA-binding activity induced by tumor factors. In the absence of Stat 3 activity, tube formation process induced by the tumor soluble factors was significantly inhibited. A significant reduction in the number of tubes per well was observed for each of the 3 concentrations of tumor supernatant used; additionally, blocking Stat 3 with CPA7 significantly inhibited basal-level tube formation by the cultured ECs without stimulation by tumor factors (Figure 5A). To confirm the results generated by the small-molecule Stat 3 inhibitor, we used an siRNA approach to knock down Stat3 gene expression in ECs. Transfecting a mouse Stat 3 siRNA into the ECs reduced the levels of phospho-Stat 3 protein (Figure 5B). While conditioned medium with the tumor supernatant stimulated EC tube formation, knocking down Stat3 in ECs effectively inhibited tumor factor-induced tube formation (Figure 5B). 
A
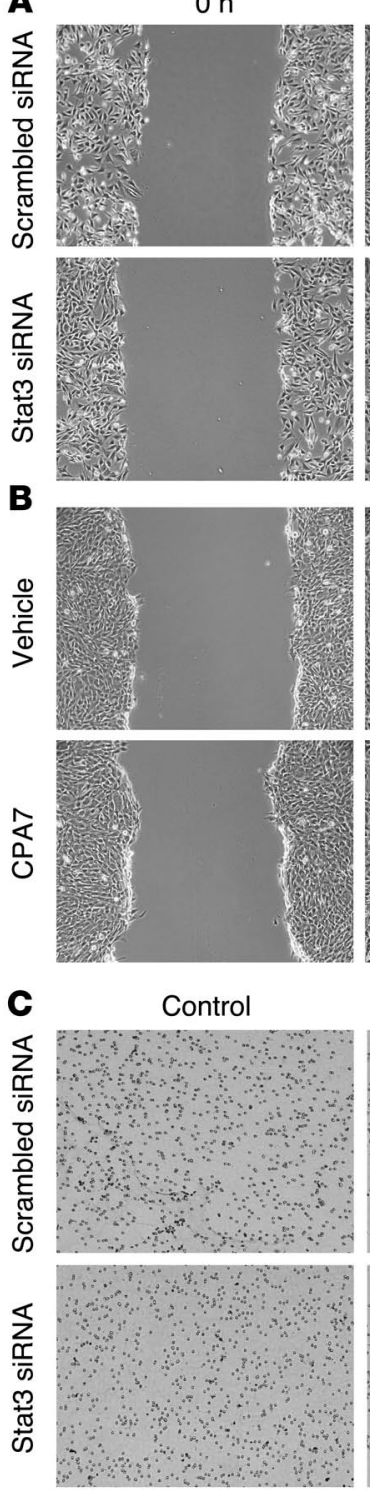

$16 \mathrm{~h}$
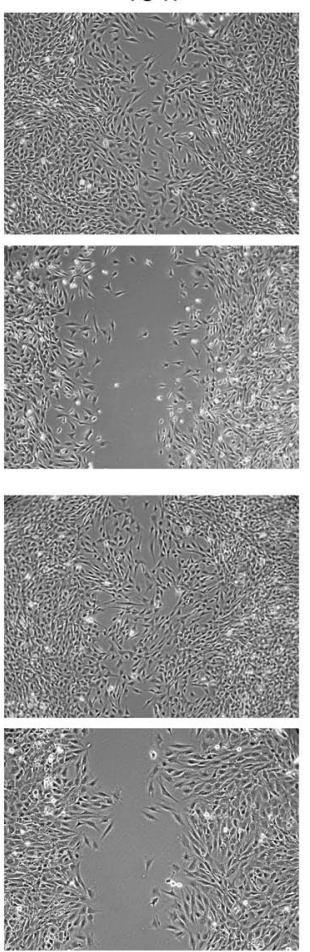

Tumor spnt
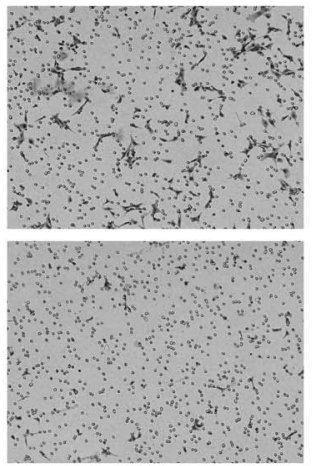
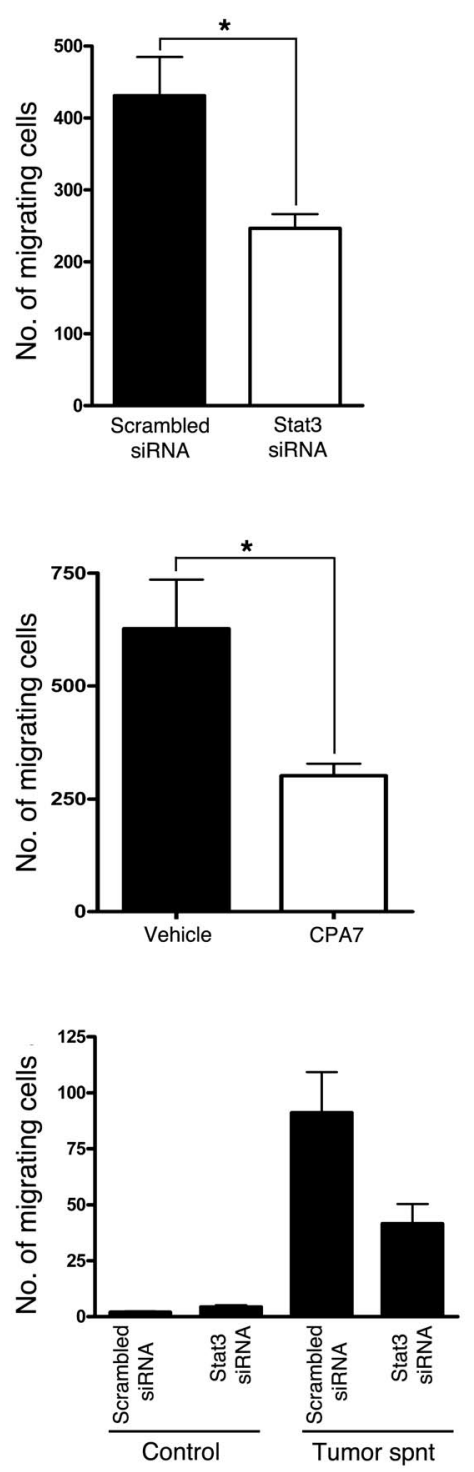

\section{Figure 6}

Stat3 activity is required for tumor factorinduced migration of ECs. (A) Representative microphotographs of wound healing assay of ECs transfected with scrambled or Stat3 siRNA and exposed to $10 \%$ tumor-conditioned media at the beginning and end of incubation ( 0 and $16 \mathrm{~h}$, respectively). Quantification of cells that migrated into the wound is also shown. (B) Representative microphotographs of wound healing assay of ECs treated with vehicle or Stat3 inhibitor CPA7 and exposed to $10 \%$ tumor-conditioned media at 0 and $16 \mathrm{~h}$ of incubation. Quantification of cells that migrated into the wound is also shown. (C) Representative microphotographs of Transwell migration assay inserts of ECs transfected with scrambled or Stat3 siRNA after $6 \mathrm{~h}$ of migration toward control medium (RPMI medium; 1\% FBS) or $10 \%$ tumor-conditioned media, stained with Harris hematoxylin solution. Quantification of cells that migrated toward control and tumor-conditioned media is also shown. Data are mean \pm SEM of 3 independent experiments; for each, 4 images were analyzed. ${ }^{*} P<0.05$. Original magnification, $\times 100$.
Intrinsic Stat 3 activity positively regulates migration of ECs. The vascularization process requires activation of many cellular and subcellular mechanisms, among which are migration and motility of ECs. To test whether Stat 3 activation in ECs contributes to migration and/or mobility of ECs, we performed wound healing assays. We blocked Stat 3 using Stat 3 siRNA and the small-molecule Stat 3 inhibitor CPA7, which caused a significant reduction in the number of cells that migrated into the wounding area (Figure 6, A and B). The same inhibitory effect on EC migration and mobility by Stat 3 blockade was observed using Transwell migration assay. Tumor-soluble factors released by the C4 melanoma cells with high Stat 3 activity induced robust migration of ECs. Blocking Stat 3 activity in ECs by siRNA significantly reduced the number of cells migrating toward the tumor-conditioned medium (Figure 6C).

Stat 3 regulates induction of tumor angiogenesis by myeloid cells in vivo. To determine whether tumor angiogenesis mediated by tumorinfiltrating myeloid cells directly requires Stat3, we performed in vivo angiogenesis assays. Because our in vitro results indicated that tumor-derived factors are required to induce and maintain angiogenic potential of myeloid cells (Figure 2A), we implanted Matrigel plugs containing B16 tumor cells admixed with either Stat3 $3^{+/+}$ or Stat $3^{-/-} \mathrm{CD} 11 \mathrm{~b}^{+} \mathrm{CD} 11 \mathrm{c}^{-}$myeloid cells. The recipient mice were Stat 3 deficient, in order to avoid the infiltration of the Matrigel by host-derived Stat3-positive myeloid cells. At $6 \mathrm{~d}$ after implantation, Matrigel plugs were harvested to measure hemoglobin content, which is indicative of neovascularization. Matrigel plugs containing the $\mathrm{Stat}^{+/+}$myeloid cells showed a significant increase in tumor angiogenesis, whereas those with Stat $3^{-/-}$myeloid cells did not have significantly increased vascularization compared with Matrigel plugs containing B16 tumor cells alone (Figure 7A and Tables 1 and 2). Results of immunofluorescence analysis of Matrigel sections using CD31 antibody support the results from hemoglobin content assays; namely, there was more microvessel density in the Matrigel plugs containing Stat $3^{+/+}$myeloid cells (Figure 7B). Data generated from the in vivo angiogenesis assays recapitulated 
A

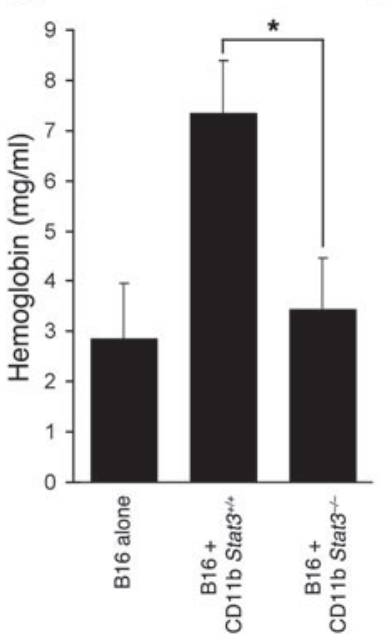

B $\mathrm{B} 16+\mathrm{CD} 11 \mathrm{~b} \mathrm{Stat3}{ }^{+/+}$
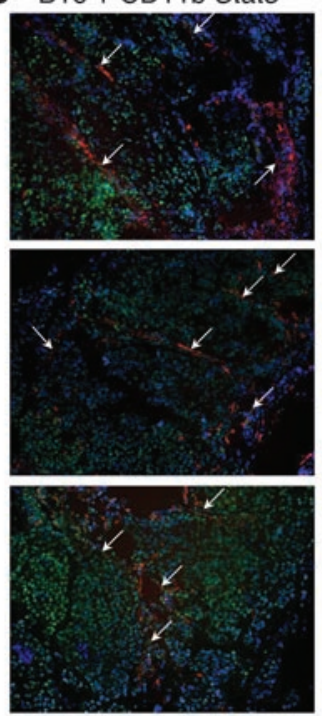

B16 + CD11b Stat3-
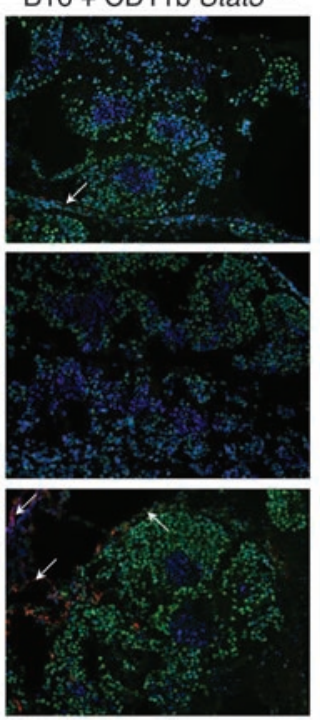

Figure 7

Stat3 is critical for myeloid cell-induced tumor angiogenesis by in vivo. (A) Matrigel plugs containing a mixture of B16 melanoma cells and CD11b+CD11 $\mathrm{c}^{-}$myeloid cells isolated from spleens of tumor-bearing mice were implanted into mice with Stat3 ${ }^{-1-}$ hematopoietic system. Both $\mathrm{Stat}^{+/+}$and $\mathrm{Stat}^{-{ }^{--}}$myeloid cells were used for the Matrigel assays. Plugs were harvested for hemoglobin content measurement $6 \mathrm{~d}$ after implanting in vivo. Data are mean \pm SEM of 3 independent experiments combined. ${ }^{*} P<0.05$ (see Methods, Table 1, and Table 2 for detailed statistical analysis). (B) Representative microphotographs of Matrigel plug frozen sections stained with CD31 (red) and pY705-Stat3 (green) antibodies. Arrows indicate newly formed vessels. Original magnification, $\times 100$. (C) Stat3 activity allows multidirectional crosstalk in the tumor stroma. Stat3 activity in tumor cells propagates, through Stat3-regulated factors (circles), to myeloid cells and ECs, and Stat3 activity in myeloid cells can further impact Stat3 activity in ECs, contributing to tumor angiogenesis.
C

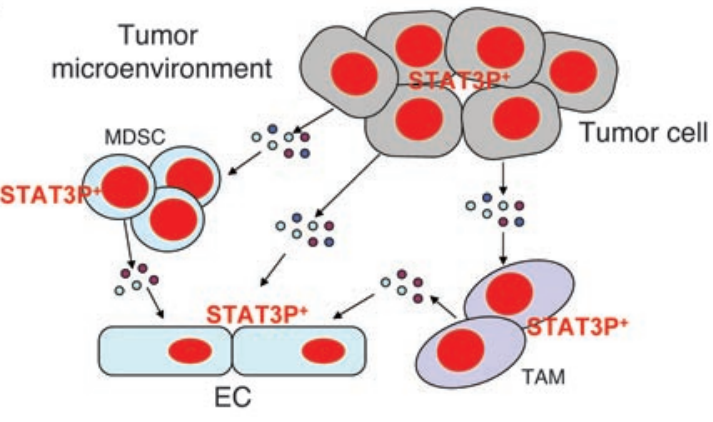

what we observed in vitro: Stat 3 activity in tumor cells propagated to myeloid cells and ECs, and Stat 3 activity in myeloid cells further enhanced Stat 3 activity in ECs, contributing to tumor angiogenesis via elevated production of angiogenic factors induced by constitutive Stat 3 activation (Figure 7C).

\section{Discussion}

The importance of TAMs in promoting cancer progression through angiogenesis and/or invasion has been well documented $(16,17,37$, 40). However, the underlying molecular mechanisms that define their carcinogenic characteristics remain to be fully explored. More recent studies have also shown that bone marrow-derived leucocytes, including $\mathrm{Gr}^{+} \mathrm{CD} 11 \mathrm{~b}^{+} \mathrm{MDSCs}$, contribute to tumor angiogenesis by producing MMP9 and by directly incorporating into tumor endothelium $(9,37)$. Furthermore, in the absence of TGF- $\beta$ signaling, mammary tumor cells recruit $\mathrm{Gr} 1^{+} \mathrm{CD} 11 \mathrm{~b}^{+}$MDSCs, leading to tumor metastasis (13). The data presented in the present study demonstrate that constitutive activation of Stat 3 in both TAMs and MDSCs was important for myeloid cell-mediated tumor angiogenesis. In support of data previously obtained using mouse systems, our limited analysis of human breast cancer tissues indicated that activation of Stat3 is not only in tumor cells, but also in tumor stromal myeloid cells surrounding ECs. Further experiments involving far more human tumor specimens with different levels of Stat 3 activity are highly desirable. We also show that in tumor-associated myeloid cells, Stat3 upregulates expression angiogenic genes, including VEGF, bFGF, IL-1 $\beta$, MMP9, CCL2, and CXCL2. Neutralizing VEGF and/or bFGF led to partial but significant reduction of tumor-associated myeloid cell-induced tube formation. Akin to the relationship between tumor cells and tumor-associated DCs and other immune effector cells such as neutrophils and NK cells in tumor immune evasion $(3,23)$, propagation of Stat 3 activity from tumor-associated myeloid cells to ECs is important for EC tumorigenic activity. Our findings in growing tumors and in the simulated tumor milieu that Stat3 activation in ECs was important for tumor angiogenesis are in agreement with previous reports indicating a requirement of Stat3 activity in EC tube formation and migration upon VEGF stimulation in vitro $(41,42)$. Nevertheless, the current study involves the tumor microenvironment with multiple interacting factors.

Our present results, together with several other published reports $(3,23)$, illustrate a fundamental mechanism that allows multidirectional crosstalk among diverse tumor stromal cells with tumor cells, promoting tumor progression. This is achieved, at least in part, by a Stat 3 feed-forward mechanism mediated by Stat 3 downstream gene products, including (but not limited to) VEGF, bFGF, IL-10, IL-23, and HGF. In support of the importance of tumorinfiltrating myeloid cells in enhancing Stat 3 activity in tumor cells is the observation that tumor cells purified from tumors grown in mice with Stat3-/- myeloid cells have reduced phospho-Stat3 (M. Kortylewski and H. Yu, unpublished observations). The fact that some of these factors activate other critical oncogenic pathways underscores the importance of participation of diverse signaling pathways for myeloid cell-mediated tumorigenesis and/or angiogenesis. VEGF, bFGF, and HGF, for example, are also activa- 
Table 1

Least square means for each treatment

$\begin{array}{lccc}\text { Treatment } & \boldsymbol{n} & \text { Least square mean } & \mathbf{9 5 \%} \mathbf{~ C l} \\ \text { B16 cells only } & 5 & 2.855 & 0.636 \text { to } 5.074 \\ \text { B16 and Stat3-/- CD11b } & 6 & 3.431 & 1.363 \text { to } 5.499 \\ \text { B16 and Stat3+/ CD11b+ } & 6 & 7.349 & 5.281 \text { to } 9.417\end{array}$

$\mathrm{Cl}$, confidence interval.

tors of the Akt pathway, and IL-23 activates not only Stat3, but also Stat5 (43). The critical roles of Akt and Stat5 in promoting cancer, which are partially mediated via angiogenesis, are well established $(44,45)$. At this point, the relative contributions to tumor angiogenesis and the relative levels of angiogenic factors produced by tumor cells versus tumor-associated myeloid cells are unknown. However, it is likely that the crosstalk among tumor cells, myeloid cells, and ECs is essential for tumor angiogenesis, and each type of the cells may contribute differently at various stages of tumor development.

Stat 3 is known as a negative regulator of granulopoiesis and ablating Stat 3 in hematopoietic cells results in increased numbers of granulocytes $(3,33,46)$. Because the surface markers used for the identification of MDSCs are common for several populations of myeloid and granulocyte cells, it is not feasible to unequivocally determine the numbers of MDSCs using immunohistochemical staining of tumor tissues. However, as shown in the current study, Stat $3^{-/-}$tumor-associated $\mathrm{Gr} 1^{+}$myeloid cells no longer supported tumor vascularization. We note that our experiments were performed in an isolated environment of Matrigel plugs containing tumor cells and myeloid cells in order to demonstrate that Stat3 can affect MDSC-mediated tumor angiogenesis. A reduction in angiogenesis associated with Stat $3^{-/-}$MDSCs is not likely to cause obvious tumor growth inhibition if is not supported by $\mathrm{T}$ celldependent antitumor immune responses in mice with a Stat3-/myeloid compartment (3). Nevertheless, while the reduction in angiogenesis by $\mathrm{Stat}^{-/-}$MDSCs was not sufficient to cause tumor regression in the aggressive B16 tumor model, it can still contribute to $\mathrm{T}$ cell-mediated antitumor effects by affecting the state of tumor vasculature.

Tumor-infiltrating myeloid cells undergo a fundamental functional change as a result of Stat 3 ablation: the displacement of several key angiogenic factors in MDSCs and macrophages with antiangiogenic mediators released by reactivated DCs. This is reminiscent of the dual characteristics of macrophages; the welldescribed M1 is pro-antitumor immune responses, and M2 is associated with inflammation-mediated carcinogenesis. Whether macrophages are M1 are M2 depends on what they produce - Th1 immune mediators, such as IFNs and IL-12, or immunosuppressive, angiogenic, and/or growth factors, including IL-10, VEGF, bFGF, IL-23, IFN- $\beta$, and MMP9. Importantly, many of the Th1 immunostimulatory molecules, including IFN- $\beta$, IFN- $\gamma$, IP- 10 , and IL-12, are also antiangiogenic factors. In parallel, several angiogenic factors, including VEGF and IL-23, play key roles in immunosuppression. The ability of Stat 3 to contribute to modulation of these factors - inhibiting immunostimulating and antiangiogenic factors while upregulating immunosuppressive and angiogenic factors - highlights the importance of oncogenic signaling in inflammation-induced carcinogenesis, further underscoring the point that the oncogenic process is what converts the mediators of our immune system from mounting antitumor immunity to immune evasion and carcinogenesis.

\section{Methods}

Cells and cell culture conditions. The B16 cell line was obtained originally from American Type Culture Collection and has been cultured in our laboratory for more than 10 years. The highly metastatic clone of K1735 melanoma (C4) and EC lines derived from prostate and colon were kindly provided by S. Huang and J. Fidler (M.D. Anderson Cancer Center, Houston, Texas). These cell lines were maintained in RPMI medium supplemented with 5\%-10\% FBS and $100 \mathrm{U} / \mathrm{ml}$ penicillin/streptomycin.

Animals. Stat $3^{\text {flox }}$ mice were provided by S. Akira (Osaka University, Suita, Osaka, Japan) and K. Takeda (Kyushu University, Fukuoka, Japan). Mouse care and experimental procedures were performed under pathogen-free conditions in accordance with established institutional guidance and approved protocols from the Institutional Animal Care and Use Committee of Beckman Research Institute at City of Hope National Medical Center.

Cell transfection and treating cells with Stat 3 small molecule inhibitor. To suppress Stat 3 expression, siRNA transfections were carried out using Lipofectamine 2000 (Invitrogen). To block Stat3 signaling with the Stat3 small-molecule inhibitor CPA7, tumor cells and ECs were treated with CPA7 at $20 \mu \mathrm{M}$. The supernatants were collected, or in vitro experiments were performed, $24 \mathrm{~h}$ after CPA7 treatment. The sequences for mouse Stat 3 siRNA and negative control (scrambled sequence) were $5^{\prime}$-UUAGCCCAUGUGAUCUGACACCCUGAAT- $3^{\prime}$ and $5^{\prime}$-UCCAAGUAGAUUCGACGGCGAAGUG-3', respectively. At $24 \mathrm{~h}$ after transfection, the cells were used for tube formation or migration assays. For RNA and protein analyses, cells were processed $48 \mathrm{~h}$ after transfection.

Tube formation assay. ECs $\left(5 \times 10^{4}\right.$ per 0.2 - $\mathrm{ml}$ well of a 48 -well plate $)$ were seeded on neutralized collagen $(1.2 \mathrm{mg} / \mathrm{ml}$; BD Biosciences) in RPMI medium with $1 \%$ FBS with or without tumor supernatant at various concentrations for $16 \mathrm{~h}$. The cells were fixed in $4 \%$ paraformaldehyde (USB) for $10 \mathrm{~min}$ in RT, washed, and analyzed under an inverted light microscope (Nikon). All tube-like structures were counted for each well; only closed networks of vessel-like tubes were counted. In some experiments ECs were coincubated with different subsets of myeloid cells (1:1 ratio, each $4.5 \times 10^{4}$ per $0.2-\mathrm{ml}$ well of a 48 -well plate). Myeloid cells were sorted from tumors or spleens of tumor-bearing mice. CD $11 b^{+}$cells were first enriched using EasySep kit (StemCell Technologies), then sorted using a MoFlo sorter (Dako) after labeling with antibodies against extracellular markers (CD11b, CD11c, and Gr1; all BD Biosciences). The purity achieved was greater than $98 \%$.

For antibody neutralization studies, myeloid cells were sorted by flow cytometry sorter from B16 melanoma tumors or enriched with EasySep kit (StemCell Technologies) from spleens and then coincubated with ECs on collagen matrix in the presence of neutralizing antibodies against VEGF $(5 \mu \mathrm{g} / \mathrm{ml}$; R\&D Systems) and bFGF $(5 \mu \mathrm{g} / \mathrm{ml}$; Abcam Inc.), alone or in combination. IgG antibody at the same concentration was used as control.

In vivo Matrigel angiogenesis assay. Mice with or without inducible ablation of Stat3 in hematopoietic cells, as described previously (3), were used.

\section{Table 2}

Pairwise comparisons between groups

\begin{tabular}{|c|c|c|}
\hline Treatment & t statistic & $P A$ \\
\hline B16 and Stat3 ${ }^{-/-}$CD11b+ vs. B16 & 0.42 & 0.91 \\
\hline B16 and Stat $3^{++}$CD11b+ vs. B16 & 3.31 & 0.02 \\
\hline $\begin{array}{l}\text { B16 and Stat3 } 3^{++} \text {CD11b } b^{+} \text {vs. } \\
\text { B16 and Stat3-/- CD11b b+ }\end{array}$ & 3.00 & 0.03 \\
\hline
\end{tabular}

ATreatments vs. control. 
CD $11 \mathrm{~b}^{+}$cells $\left(5 \times 10^{5}\right)$ were enriched from spleens (greater than $90 \%$ purity) after removal of CD11 $\mathrm{c}^{+}$cells using positive selection EasySep kits (StemCell Technologies) and mixed with $5 \times 10^{4}$ B16 melanoma cells in $600 \mu \mathrm{l}$ growth factor-reduced Matrigel (BD Biosciences) and injected into Stat3 ${ }^{-/-}$ mice. Matrigel plugs were dissected $6 \mathrm{~d}$ later and either analyzed for hemoglobin content by colorimetry using Drabkin reagent (Sigma-Aldrich) or frozen and analyzed by immunofluorescent staining.

Flow cytometry. Cells suspensions from spleen and tumor tissues were prepared as described previously (3) and stained with different combinations of fluorochrome-coupled antibodies to CD11c, CD11b, Gr1, pY705-Stat3, and VEGF (BD Biosciences). Fluorescence data were collected on a FACSCalibur (BD) and analyzed using FlowJo software (version 7.2.4; Tree Star).

Wound healing and transwell migration assays. ECs were grown on 6-cm plates until $90 \%$ confluence, and 2 scratches with a tip were done along the plate. Cells were rinsed and exposed overnight to $10 \%$ tumor-conditioned media.

At the beginning and end of the experiments, 4 images of the wound were taken, and the number of cells migrating into the wound was counted using ImageJ software (http://rsb.info.nih.gov/ij/). In Transwell migration assay, collagen-coated inserts with $8 \mu \mathrm{m}$ pore size (Corning-Costar) were used. Cells $\left(15 \times 10^{5}\right)$ resuspended in RPMI medium were placed in the top chamber of the insert, and the bottom well was filled with RPMI medium with or without $10 \%$ tumor supernatant added. After $6 \mathrm{~h}$, the inserts were removed, and the inner side was whipped with cotton swaps and stained with Harris hematoxylin solution (Sigma-Aldrich). After washing, filters were cut out, mounted on microscope slides, and analyzed. For each filter, 4 images covering the majority of the sample were collected, and the cells were counted using ImageJ software.

RNA isolation and real-time PCR analysis. Total RNA was isolated using RNeasy Kit (Qiagen) according to the manufacturer's protocol. Reverse transcriptions were done using iScript cDNA Synthesis Kit (Bio-Rad), and real-time PCR reactions were performed using iQ SYBR Green supermix (Bio-Rad) on DNA Engine thermal cycler equipped with Chromo4 detector (Bio-Rad). For gene expression analysis of Stat3, VEGF, IL-1 1 , MMP9, CCL2, and CXCL2, with 18S rRNA or GAPDH housekeeping genes as internal controls, we used mouse-specific primer sets purchased from SuperArray.

Cell lysis and Western blot analysis. Cell lysates were prepared as described previously (47) and clarified by centrifugation, and protein concentrations of supernatants were determined by Bio-Rad protein assay. Equivalent amounts of total cellular proteins were separated by SDS plus $10 \%$ PAGE, transferred onto nitrocellulose membranes, probed with the respective antibodies, and detected for signals using horseradish peroxidase-conjugated secondary antibodies by enhanced chemiluminescence (Pierce Biotechnology).

$b F G F$ detection. To detect bFGF, myeloid cells were isolated from spleens, exposed to tumor-conditioned medium, and incubated for $4 \mathrm{~h}$ in the presence of $10 \mu \mathrm{g} / \mathrm{ml}$ brefeldin A (Sigma-Aldrich). Collected cells were incubated in hypotonic buffer and sonicated, and cytoplasmatic fractions were analyzed using cytokine bead array (Invitrogen) and Luminex system according to the manufacturer's protocol.

EMSA. EMSA to detect Stat3 $\beta$ DNA binding was performed essentially as previously described (48). Briefly, nuclear extracts (1-8 $\mu \mathrm{g}$ of total protein), normalized for protein concentration, were incubated with the ${ }^{32} \mathrm{P}$-radiolabled high-affinity Sis-Inducible Element (hSIE) oligonucleotide probe. Protein-DNA complexes were resolved by $5 \%$ nondenatured PAGE, and specific STAT/DNA complexes were detected by autoradiography.

Immunohistochemistry and immunofluorescence. Tumor tissues were formalin fixed and paraffin embedded. Sections of $3 \mu \mathrm{m}$ were deparaffinized and stained with $\mathrm{pY}^{705}$-Stat3 antibody (1:50; Santa Cruz Biotechnology Inc.). Archival human breast carcinoma tissue arrays from an anonymous group of patients were provided by the Pathology Core of City of Hope Comprehensive Cancer Center. Sections were deparaffinized and stained with rabbit $\mathrm{pY}^{705}$-Stat 3 antibody and mouse anti-CD68 (1:40; AbD Serotec), a marker for human macrophages and monocytes. For fluorescent detection, secondary antibodies were used (Alexa Fluor 488-labeled goat anti-rabbit and Alexa Fluor 555-labeled goat anti-mouse; 1:100, both Invitrogen).

For immunofluorescence staining in coincubation experiments before trypsinization, ECs were labeled with CellTracker OrangeCMTMR reagent (Invitrogen) at the final concentration of $5 \mu \mathrm{M}$, and then washed 3 times. ECs alone or mixed 1:5 with C4 melanoma cells (total $8 \times 10^{5}$ cells) were seeded on round coverslips placed in 24 -well plates. After overnight culture, cells were fixed with methanol at $-20^{\circ} \mathrm{C}$, permeabilized, blocked with $1 \%$ BSA solution, and incubated overnight with $\mathrm{PY}^{705}$-Stat 3 antibody (1:50; Santa Cruz Biotechnology Inc.). The next day, the coverslips were washed, and Alexa Fluor 488-labeled goat anti-rabbit secondary antibody was applied for $1 \mathrm{~h}$ (1:100; Invitrogen). After washing, coverslips were mounted with Vectashield medium containing DAPI (Vector Laboratories).

For immunofluorescent staining of frozen Matrigel plugs, sections were fixated in $2 \%$ paraformaldehyde and permeabilized in methanol. After blocking in PBS containing $10 \%$ goat serum and $2.5 \%$ mouse serum, slides were incubated overnight with CD31 (1:50; BD Biosciences) and pY705-Stat3 (1:50; Santa Cruz Biotechnology Inc.) antibodies. The next day, the slides were washed, and Alexa Fluor 488-labeled goat anti-rabbit and Alexa Fluor 555-labeled goat anti-rat secondary antibodies were applied for $1 \mathrm{~h}$ (1:100; Invitrogen).

Statistics. For data obtained from in vivo Matrigel assays, a general linear model was used to analyze the association between the outcome and the treatment effect adjusting for experimental effects. Normality and equal variance assumption were checked, and requirements were satisfied. To reduce the type I error, we used the Tukey's adjustment for the results. A $P$ value less than 0.05 was considered significant.

\section{Acknowledgments}

We thank staff members of the Clinical Immunobiology Correlative Studies Laboratory, the Analytic Microscopy Core, the Pathology Research Core, and the Animal Research Center at City of Hope National Medical Center for technical assistance. We also acknowledge Xiwei Wu of the Information Science Department at City of Hope National Medical Center for statistical analysis. We are indebted to S. Akira and K. Takeda for providing Stat $3^{f l o x}$ mice. This work was supported by NIH and Harry Lloyd Charitable Trust grants to H. Yu.

Received for publication January 31, 2008, and accepted in revised form July 23, 2008.

Address correspondence to: Hua Yu, Beckman Research Institute, City of Hope National Medical Center, 1500 E. Duarte Road, Duarte, California 91010, USA. Phone: (626) 471-7238; Fax: (626) 256-8708; E-mail: hyu@coh.org.

Maciej Kujawski and Marcin Kortylewski contributed equally to this work.

Heidi Kay's present address is: Paradigm Biotech, Tampa, Florida, USA.
1. Dunn, G.P., Bruce, A.T., Ikeda, H., Old, L.J., and Schreiber, R.D. 2002. Cancer immunoediting: from immunosurveillance to tumor escape. Nat. Immunol. 3:991-998.
2. Pardoll, D. 2003. Does the immune system see tumors as foreign or self? Annu. Rev. Immunol. 21:807-839.

3. Kortylewski, M., et al. 2005. Inhibiting Stat3 signal- ing in the hematopoietic system elicits multicomponent antitumor immunity. Nat. Med. 11:1314-1321.

4. Yu, P., et al. 2005. Intratumor depletion of CD4+ cells unmasks tumor immunogenicity leading 
to the rejection of late-stage tumors. J. Exp. Med. 201:779-791.

5. Curiel, T.J., et al. 2004. Specific recruitment of regulatory $T$ cells in ovarian carcinoma fosters immune privilege and predicts reduced survival. Nat. Med. 10:942-949.

6. Ghiringhelli, F., et al. 2005. Tumor cells convert immature myeloid dendritic cells into TGF-betasecreting cells inducing CD4+CD25+ regulatory T cell proliferation. J. Exp. Med. 202:919-929.

7. Melani, C., Chiodoni, C., Forni, G., and Colombo, M.P. 2003. Myeloid cell expansion elicited by the progression of spontaneous mammary carcinomas in c-erbB-2 transgenic BALB/c mice suppresses immune reactivity. Blood. 102:2138-2145.

8. Pan, P.Y., et al. 2008. Reversion of immune tolerance in advanced malignancy: modulation of myeloidderived suppressor cell development by blockade of stem-cell factor function. Blood. 111:219-228.

9. Yang, L., et al. 2004. Expansion of myeloid immune suppressor $\mathrm{Gr}+\mathrm{CD} 11 \mathrm{~b}+$ cells in tumor-bearing host directly promotes tumor angiogenesis. Cancer Cell. 6:409-421.

10. Allavena, P., Sica, A., Solinas, G., Porta, C., and Mantovani, A. 2008. The inflammatory micro-environment in tumor progression: the role of tumor-associated macrophages. Crit. Rev. Oncol. Hematol. 66:1-9.

11. Melani, C., Sangaletti, S., Barazzetta, F.M., Werb, Z., and Colombo, M.P. 2007. Amino-biphosphonatemediated MMP-9 inhibition breaks the tumor-bone marrow axis responsible for myeloid-derived suppressor cell expansion and macrophage infiltration in tumor stroma. Cancer Res. 67:11438-11446.

12. Voronov, E., et al. 2003. IL-1 is required for tumor invasiveness and angiogenesis. Proc. Natl. Acad. Sci. U. S. A. 100:2645-2650

13. Yang, L., et al. 2008. Abrogation of TGFbeta signaling in mammary carcinomas recruits $\mathrm{Gr}-1+\mathrm{CD} 11 \mathrm{~b}+$ myeloid cells that promote metastasis. Cancer Cell. 13:23-35.

14. Bunt, S.K., et al. 2007. Reduced inflammation in the tumor microenvironment delays the accumulation of myeloid-derived suppressor cells and limits tumor progression. Cancer Res. 67:10019-10026.

15. Bunt, S.K., Sinha, P., Clements, V.K., Leips, J., and Ostrand-Rosenberg, S. 2006. Inflammation induces myeloid-derived suppressor cells that facilitate tumor progression. J. Immunol. 176:284-290.

16. Condeelis, J., and Pollard, J.W. 2006. Macrophages: obligate partners for tumor cell migration, invasion, and metastasis. Cell. 124:263-266.

17. Pollard, J.W. 2004. Tumour-educated macrophages promote tumour progression and metastasis. Nat. Rev. Cancer. 4:71-78.

18. Sica, A., and Bronte, V. 2007. Altered macrophage differentiation and immune dysfunction in tumor development. J. Clin. Invest. 117:1155-1166.

19. Yu, H., and Jove, R. 2004. The STATs of cancer new molecular targets come of age. Nat. Rev. Cancer. 4:97-105.

20. Yu, C.L., et al. 1995. Enhanced DNA-binding activity of a Stat3-related protein in cells transformed by the Src oncoprotein. Science. 269:81-83.

21. Gao, S.P., et al. 2007. Mutations in the EGFR kinase domain mediate STAT3 activation via IL-6 production in human lung adenocarcinomas. J. Clin. Invest. 117:3846-3856.

22. Nabarro, S., et al. 2005. Coordinated oncogenic transformation and inhibition of host immune responses by the PAX3-FKHR fusion oncoprotein. J. Exp. Med. 202:1399-1410.

23. Wang, T., et al. 2004. Regulation of the innate and adaptive immune responses by Stat- 3 signaling in tumor cells. Nat. Med. 10:48-54.

24. Kinjyo, I., et al. 2006. Loss of SOCS3 in T helper cells resulted in reduced immune responses and hyperproduction of interleukin 10 and transforming growth factor-\{beta\}1. J. Exp. Med. 203:1021-1031.

25. Niu, G., et al. 2002. Constitutive Stat 3 activity upregulates VEGF expression and tumor angiogenesis. Oncogene. 21:2000-2008.

26. Sumimoto, H., Imabayashi, F., Iwata, T., and Kawakami, Y. 2006. The BRAF-MAPK signaling pathway is essential for cancer-immune evasion in human melanoma cells. J. Exp. Med. 203:1651-1656.

27. Yu, H., Kortylewski, M., and Pardoll, D. 2007. Crosstalk between cancer and immune cells: role of STAT3 in the tumour microenvironment. Nat. Rev. Immunol. 7:41-51.

28. Wei, D., et al. 2003. Stat3 activation regulates the expression of vascular endothelial growth factor and human pancreatic cancer angiogenesis and metastasis. Oncogene. 22:319-329.

29. Xie, T.X., et al. 2006. Activation of stat 3 in human melanoma promotes brain metastasis. Cancer Res. 66:3188-3196.

30. Dechow, T.N., et al. 2004. Requirement of matrix metalloproteinase- 9 for the transformation of human mammary epithelial cells by Stat3-C. Proc. Natl. Acad. Sci. U. S. A. 101:10602-10607.

31. Nakao, S., et al. 2005. Infiltration of COX-2expressing macrophages is a prerequisite for IL-1 beta-induced neovascularization and tumor growth. J. Clin. Invest. 115:2979-2991.

32. Porta, C., et al. 2007. Tumor promotion by tumorassociated macrophages. Adv. Exp. Med. Biol. 604:67-86.

33. Lee, C.K., et al. 2002. STAT3 is a negative regulator of granulopoiesis but is not required for G-CSFdependent differentiation. Immunity. 17:63-72.
34. Wilhelm, S.M., et al. 2004. BAY 43-9006 exhibits broad spectrum oral antitumor activity and targets the RAF/MEK/ERK pathway and receptor tyrosine kinases involved in tumor progression and angiogenesis. Cancer Res. 64:7099-7109.

35. Zeng, Q., et al. 2005. Crosstalk between tumor and endothelial cells promotes tumor angiogenesis by MAPK activation of Notch signaling. Cancer Cell. 8:13-23.

36. Balkwill, F. 2004. Cancer and the chemokine network. Nat. Rev. Cancer. 4:540-550.

37. Jodele, S., et al. 2005. The contribution of bone marrow-derived cells to the tumor vasculature in neuroblastoma is matrix metalloproteinase- 9 dependent. Cancer Res. 65:3200-3208.

38. Langley, R.R., et al. 2003. Tissue-specific microvascular endothelial cell lines from $\mathrm{H}-2 \mathrm{~K}(\mathrm{~b})$-tsA58 mice for studies of angiogenesis and metastasis. Cancer Res. 63:2971-2976.

39. Burdelya, L., et al. 2005. Stat 3 activity in melanoma cells affects migration of immune effector cells and nitric oxide-mediated antitumor effects. J. Immunol. 174:3925-3931.

40. Wyckoff, J.B., et al. 2007. Direct visualization of macrophage-assisted tumor cell intravasation in mammary tumors. Cancer Res. 67:2649-2656.

41. Bartoli, M., et al. 2003. VEGF differentially activates STAT3 in microvascular endothelial cells. FASEB J. 17:1562-1564.

42. Yahata, Y., et al. 2003. Nuclear translocation of phosphorylated STAT3 is essential for vascular endothelial growth factor-induced human dermal microvascular endothelial cell migration and tube formation. J. Biol. Chem. 278:40026-40031.

43. Hunter, C.A. 2005. New IL-12-family members: IL-23 and IL-27, cytokines with divergent functions. Nat. Rev. Immunol. 5:521-531.

44. Fang, J., Ding, M., Yang, L., Liu, L.-Z., and Jiang, B.-H. 2007. PI3K/PTEN/AKT signaling regulates prostate tumor angiogenesis. Cell. Signal. 19:2487-2497.

45. Haura, E.B., Turkson, J., and Jove, R. 2005. Mechanisms of disease: Insights into the emerging role of signal transducers and activators of transcription in cancer. Nat. Clin. Pract. Oncol. 2:315-324.

46. Panopoulos, A.D., et al. 2006. STAT3 governs distinct pathways in emergency granulopoiesis and mature neutrophils. Blood. 108:3682-3690.

47. Kortylewski, M., et al. 1999. Interleukin-6 and oncostatin M-induced growth inhibition of human A375 melanoma cells is STAT-dependent and involves upregulation of the cyclin-dependent kinase inhibitor p27/Kip1. Oncogene. 18:3742-3753.

48. Niu, G., et al. 1999. Gene therapy with dominantnegative Stat 3 suppresses growth of the murine melanoma B16 tumor in vivo. Cancer Res. 59:5059-5063. 